\title{
Increase the Lifetime of WSN by Preventing Sink Isolation using Supercluster Formation
}

\author{
R. Devika', B. Santhi' ${ }^{2}$ and T. Sivasubramanian ${ }^{3}$ \\ 1,2School of Computing, SASTRA University,Thanjavur, India; srdevika.cse@sastra.edu, b.shanthi@sastra.edu \\ ${ }^{3}$ Kings College of Engineering, Punalkulam, Pudhukkotai District, India; shivamiru@gmail.com
}

\begin{abstract}
Wireless Sensor Networks (WSN) concentrate on the essential problem of increasing the network lifetime of WSN, which are bordered by low capacity batteries 1,3 One of the fundamental egresses in WSN is maximizing the network lifetime by mitigating the Hotspot problem. It is the problem of isolation of sink node due to energy diminution of nodes most closelipped to sink1. In WSN nodes which are most adjacent to the Base Station (BS) need to relay many packets while compare with nodes far away from the sink. The outcome of this approach is these nodes deplete their energy very quickly. It results in a network partitioning and reduces the network lifetime. This paper proposes and evaluates a technique for maximizing the lifetime of WSN by preventing sink isolation due to hotspot problem. The clustering mechanism can be used to reduce the energy depletion of node near to the sink node. The cluster is formed based on distance attribute. This mechanism provides the scalability and avoids the redundancy through data collection operation. This paper will analyze the energy consumption of proposed technique using various distance measures.
\end{abstract}

Keywords: Clustering Mechanism, Data Aggregation Operation, Hotspot Problem, Network Lifetime, Sink

\section{Introduction}

Wireless Sensor Network (WSN) comprises ten to thousands tiny nodes with detecting, counting and transmitting data in wireless medium. WSN is generally used to monitor activities and report issues, such as fire, overheating etc. in a specific area or environment. Nodes transmit data returns to the sink node. Data transmission is normally from node to node towards the BS in multihop fashion. Sensor nodes are bounded in computing, communication bandwidth and power. It suffers from the hot spot problem where the nodes closest to the consumer (sink) are needed to relay many packets than the nodes beyond the base station. Hence, the lifespan of the sensor network depends on these closest nodes

Design challenges of sensor network are fault tolerance, scalability, production cost, operating environment, power consumption, data aggregations and Quality of services20. The Routing protocols play a vital role in identifying and maintaining paths in network and free from stale routes. The subtle differences between sensor and ad-hoc network is the number of nodes is more in sensor network and they are densely deployed. Due to the movement of nodes, topology of a sensor network is extremely dynamic. Nodes in sensor network have size and weight constraints along with power constraints so nodes are prone to failure. Sensor network nodes do not have global ID.

A WSN typically has little or no infrastructure. It can be classified into two major categories based on their arrangement of nodes. They are structured model and unstructured model. In Structured model, a small number of nodes are deployed in a pre-planned manner. It has lower network maintenance and lower cost. In Unstructured model large number nodes are randomly deployed. Network maintenance is difficult in case of net-

*Author for correspondence 
work connectivity and detecting failure. Figure 1 shows the client architecture. The four components of this architecture are a battery which provides power supply, sensor, a processing unit and the radio transceiver unit. The sensor unit senses the environment which is processed by the control unit and then transferred by the transceiver.

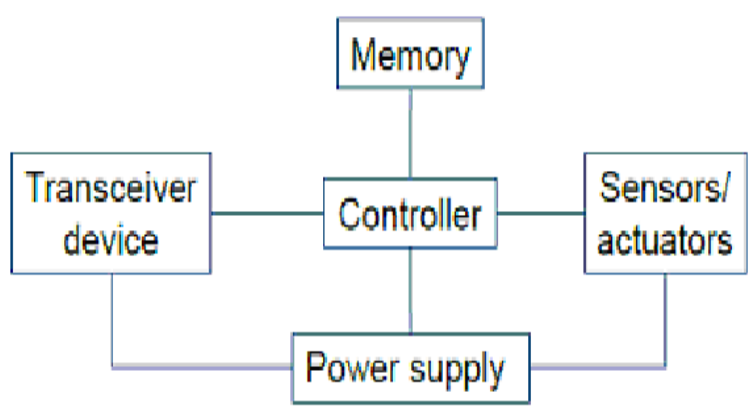

Figure 1. Node architecture.

There are three key factors ${ }^{5}$ that impact the operation of a WSN: reliability, security and performance.

\subsection{Reliability}

For any WSN, the key goal is to have reliable data transmission that is the highest probability of a packet being delivered in the presence of interference. Reliability refers to the packet error rate, error-correction coding, retransmission mechanism and frequency planning.

\subsection{Security}

Data protection in a WSN is a major challenge. Data security becomes even more significant when any security-oriented application is considered. Not only must the system ensure privacy, it must be able to authenticate data communication also. Security includes encryption and cryptographic authentication which impose major challenges in terms of the cost of power and bandwidth.

\subsection{Performance}

Performance encompasses bandwidth, data rates, and latency and packet prioritization (Qos) techniques. In fact, transmission time has a major impact on performance.

Other factors that need to be considered when developing and deploying any WSN solution are Costeffectiveness, Flexibility and Scalability, Heterogeneity,
Distributed processing, self organizing and self-healing and Energy efficiency constraints.

\subsection{Cost Effectiveness}

A key factor in the cost of wireless embedded processors is the amount of memory required. Developers of sensor networks will expect access to a range of chips or wireless microprocessors/microcontrollers with optimized memory footprints to meet the needs of a range of applications.

\subsection{Flexibility and Scalability}

WSN architecture must be flexible and adaptive enough to deploy in a wide range of usage scenarios. The number of sensors in different deployments may vary from thousands to millions. Therefore network management must be flexible enough to sustain this huge network of sensors. Scalability - primarily governed by access to media - is another challenge in a WSN.

\subsection{Heterogeneity and Distributed Processing}

The devices deployed may be of various types and need to collaborate with each other. The algorithms need to be centralized as the processing is carried out on different nodes.

\subsection{Self-Organizing and Self-Healing}

A WSN should give high reliability and predictability by applying self-organizing and self-healing mechanism to endlessly adapt to irregular conditions. The exact structure of a WSN cannot be predetermined in most situations due to variable dependencies such as location devices and radio propagation during network formation. In addition to joining a network, devices could leave, be forced to leave or potentially re-associate elsewhere within the network - all of which could cause a change in the network structure.

\subsection{Energy Efficiency Constraints}

Usually Sensor nodes are battery-driven. Sensor network are often deployed in unattended area like underwater deployment and it is very unmanageable to reload or put back the battery. Hence, energy-efficiency is a very serious concern to increase the lifetime of sensor network. 


\subsection{Large Scale Coordination and Real Time Computation}

The sensor needs to coordinate with each other to produce required results. The computation should be done quickly as new data is always being generated.

In recent year, WSN has countless challenges still it found their application in diverse fields including Disaster Relief Management, Bio diversity, and Intelligent Buildings/Bridges. The applications can be divided in three categories. Monitoring of objects, Monitoring of an area, Monitoring of both area and objects ${ }^{5}$.

The rest of this paper is orchestrated as follows. Section 2 shows routing algorithms for WSNs. In Section 3 , we introduce methods that amend the hot spot problem. Section 4 exhibits the performance analysis of our proposed method. Finally Section 5 contains the conclusion.

\section{Related Work}

The eliciting interest in WSN and the issue of modern architectural technique is the reason for studying of routing protocols. In literature many researchers have been proposed many routing protocols to extend the network lifetime by mitigating the energy consumption of nodes. These protocols can be categorized into two families: Flat routing protocol and Hierarchal protocols. Each node in flat routing forward data to another reachable node and all nodes play the same role. The advantage of this routing protocol is to choose the path with minimum energy consumption ${ }^{13}$. The main drawback of this routing protocol is failing to take the correlation between data.

Energy consumption can be reduced by doing data aggregation/data compression/data fusion operation. It will combine all data from their member and perform aggregation operation such as Average operation. It not only avoids redundant data and but also reduces the size of the packet. In the cluster routing protocol, $\mathrm{CH}$ done the data aggregation operation so that it will cut down the energy demand and get a better network lifetime ${ }^{6,7}$. Moreover in large scale WSN Scalable architectural is achieved by using cluster routing protocols. Intanagonwiw at, $\mathrm{C}$ et al ${ }^{9}$.

\subsection{Direct Diffusion}

It is a Scalable and Robust Communication Paradigm for
Sensor Networks. This is a data centric approach (i.e.) communication based on naming data not named nodes ${ }^{9,16,17}$. It requires only localized interaction between nodes. There are four main features are Interests, Gradients, Data and Reinforcement.

\subsection{Leach}

It is a hierarchical based protocol. It stands for Low Energy Adaptive Clustering Hierarchy10. Clusters are shaped dynamically and cluster head is picking out arbitrarily. Sink node will collect the data form all cluster head. It is not well suitable for large scale network.

\subsection{TEEN Protocol}

Threshold sensitive Energy Efficient sensor Network proposed by A. Manjeshwar and D.P Agrawal. Depending on the sensed data and their target application the threshold value can be changed. This varied data sent to the user immediately. It will also enhance the efficiency of wireless sensor networks ${ }^{11}$.

\subsection{SPIN}

Sensor Protocols for Information via Negotiation SPIN (Sensor Protocols for Information via Negotiation), that efficiently broadcast information among nodes ${ }^{13}$. Node will use the metadata which will eliminate the redundant data throughout the network ${ }^{12}$. Lindsey and Raghavendra ${ }^{15}$ suggest a Power Efficient Gathering in Sensor Information System called PEGASIS ${ }^{8}$, which is an enhancement over LEACH protocol. The main aim of this protocol is to extend the lifetime of network by communicating with its closest neighbor. There is no cluster formation. That is instead of multiple nodes only one node make communication with the base station ${ }^{15}$.

\subsection{HEED}

Hybrid Energy Efficient Distributed (HEED). In this protocol cluster head formation ${ }^{4}$ is based on node proximity to its neighbor and its residual energy. There is no adaptive multi-level clustering. In dynamic sensing environment it is inefficient to sense the data. During secured communication, the entire packet is encrypted at a physical layer level in order to avoid inferring traffic statistics. Hence it is unable to retrieve the source and destination addresses from the overheard packet. In such situations $\mathrm{PEACH}^{2}$ algorithm cannot be used. 


\subsection{FEAR}

A Fuzzy-Based Energy-Aware Routing Protocol. It contains three steps: Neighbor Discovery, Forward data and Energy update 22 .

\section{7 $\mathrm{ECPF}$}

An energy-aware clustering protocol. Three major techniques used in this protocol. Namely cluster head selection with non probabilistic method, fuzzy system logic technique and on need clustering technique ${ }^{18}$.

\subsection{EBMA}

Energy Balancing Cluster on Based on the cellular topology cluster is formed and energy is balanced in inter and intra cluster ${ }^{21}$.

\subsection{DDRP}

It is an efficient data driven routing protocol for WSN with mobile sink. It will reduce the topology overhead and extend the lifetime of $\mathrm{WSN}^{19}$.

Flat routing is simple protocol but it suffers a large amount of control packet overhead and lack of scalability. In recent year, researches are moved to Hierarchical routing 10,14 . The clustering routing protocol is best protocol compared with all other routing protocols. The reason behind in that it provides more scalability, consume very less energy by performing data aggregation, and finally provide robustness.

\subsubsection{More Scalability}

Scalability refers to the performance of communicating system won't be degraded when numbers of nodes will increase. In WSN, there may be thousand of nodes. It can be achieved by localizing the interaction among the communicating nodes, which can be done through hierarchical routing. Compare two flat routing it can be easily manageable.

\subsubsection{Less Energy}

In hierarchical routing Cluster head performs data aggregation and data transmission. This will lead save a great deal of energy comparable to flat and location based routing. In addition to that clustering with inter and intra cluster communication reduces the node to communicate with node present in long distance. This will also help to consume less amount of energy.

\subsubsection{More Robustness}

The topology of a WSN may change due to alternative states of sensor node from sleep mode to an active node. Hierarchal routing is highly suitable for topology control and network management. It is working well in largescale scenario compared to flat routing which is always reactive or proactive.

\section{Proposed Method}

This paper demonstrates the hotspot problem, which is the closing of consumer node due to energy depletion of nodes surrounding it. According to the multi-hop data transmission mode, the data packages are sent to the sink node through different sensors. Nodes very nearer sink node has to receive and send on data from other sensors that are remote from the sink.

The sensor which is closer to the sink, the more data it needs to forward. Therefore it requires a lot of computation and communication resources to process the data relaying work. Especially those sensors are only one hop away from the sink run through much more energy and consequently wipe out their energy quicker than the other nodes. A large portion of sensors hues depending on those "close-to-the-sink" sensors and when they die, a large amount of data cannot reach the sink, resulting in a severe downgrade of the network performance. This problem has been identified and called as a "Hotspot" problem and it can be prevented by three well known methods such as

- Direct Transmission

- Cluster Transmission

- Super Cluster Transmission

- Performance Analysis

These transmissions are done by using the technique called Euclidean distance formula Sqrt ((x1-x2)2+ (y1-y2)2) and data aggregation. Energy consumption is calculated in three transmissions. These techniques are involved in the following steps.

\subsection{Direct Transmission}

In Direct Transmission each and every node in the sensing filed directly forward the packet to base station node and energy using up of each node is calculated using the formula.

Energy calculation $=$ No. Of bits transmitted ${ }^{*}$ distance between nodes and sink. 


\subsection{Cluster Transmission}

In Cluster transmission, cluster is formed by dividing the sensor field into groups based on the attribute like distance and Cluster Head $(\mathrm{CH})$ is delegated to each consulate. Cluster head collects the data from another node and perform data Aggregation. Data aggregation greatly reduces the energy consumption of network. Cluster head itself has spent energy for performing data aggregation. So the total energy spent by the cluster head is.

Energy calculation = Aggregation of transmission energy + Aggregation of Receiving energy $+\mathrm{CH}$ to sink transmission energy.

\subsection{Super Cluster Transmission}

In Super Cluster transmission, at least two $\mathrm{CH}$ can select the one node as Coordinator node. Two Cluster Head is combined based on their energy consumption in cluster transmission. Now Coordinator sends the packet to sink so that direct communication from each $\mathrm{CH}$ to sink is prevented. Here Data aggregation is Coordinator node. Energy consumption of Coordinator node is calculated.

The super cluster generation algorithm exploits in the following steps:

Step 1: Node deployment

Step 2: Cluster Establishment using distance attribute.

Step 3: Select the Cluster Coordinator for any two Cluster Head $(\mathrm{CH})$

Step 4: Energy of SC 1 = Aggregation of transmission energy of $\mathrm{CH} 1$ and $\mathrm{CH} 2$ to coordinator+ Receiving energy of coordinator + transmission energy from coordinator to sink

Step 5: Energy of SC 2 = Aggregation of transmission energy of $\mathrm{CH} 3$ and $\mathrm{CH} 4$ to coordinator + Receiving energy of coordinate + transmission energy from coordinator to sink

Step 6: Total Energy calculation= SCF1 +SCF 2.

Step 7: Repeat the step 3 to step 6 until all the Cluster Head has Clustered Coordinator and calculate energy consumption.

\subsection{Performance Analysis}

The proposed method is evaluated and its energy consumption is compared with Direct Transmission and Cluster transmission.

\section{Experimental Setup}

Our assumption for sensor network is randomly distributed node over an area of NXN meter as shown in figure 2. Where $\mathrm{N}$ varies from multiple of $20 \mathrm{~m}$ up to $100 \mathrm{~m}$. Transmitting energy is calculated by $0.5 \mathrm{~J}$ * distances between particular node to destination. Where 0.5Joule is energy spent by the node to send data. In similar way

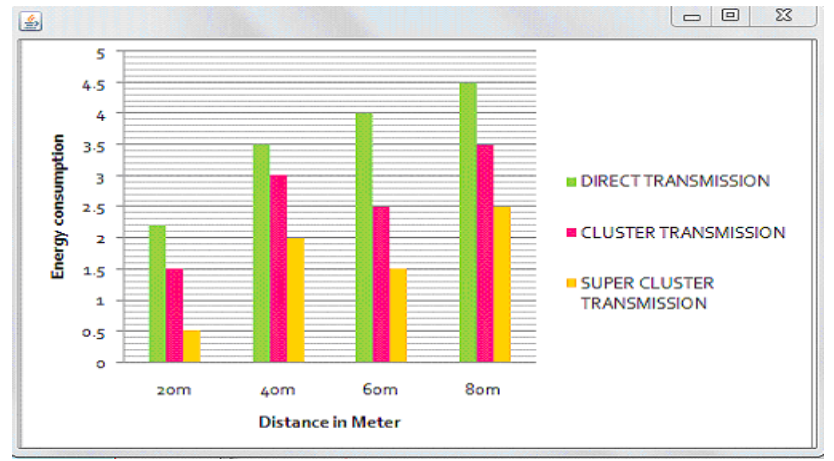

Figure 2. Nodes are randomly distributed over an area of 60X60 meter.

receiving energy for a particular node is calculated by $0.3 \mathrm{~J}^{\star}$ distances between particular node to destination. Where 0.3 Joule is the energy consumed by the node to generate the data.

In Figure 3 energy consumption is taken as the $y$ axis and three transmission methods in $\mathrm{x}$ axis. Super Cluster transmission comparatively consumes less energy than

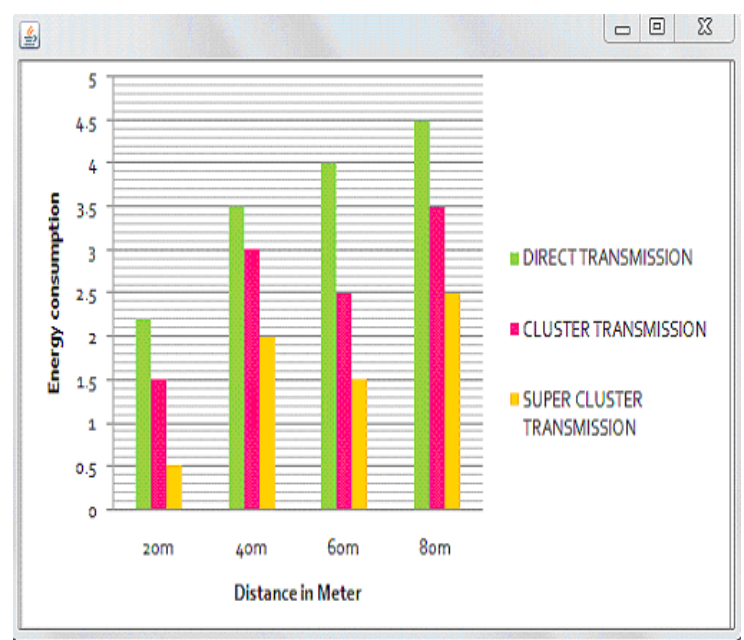

Figure 3. Total energy consumption of three transmission. 


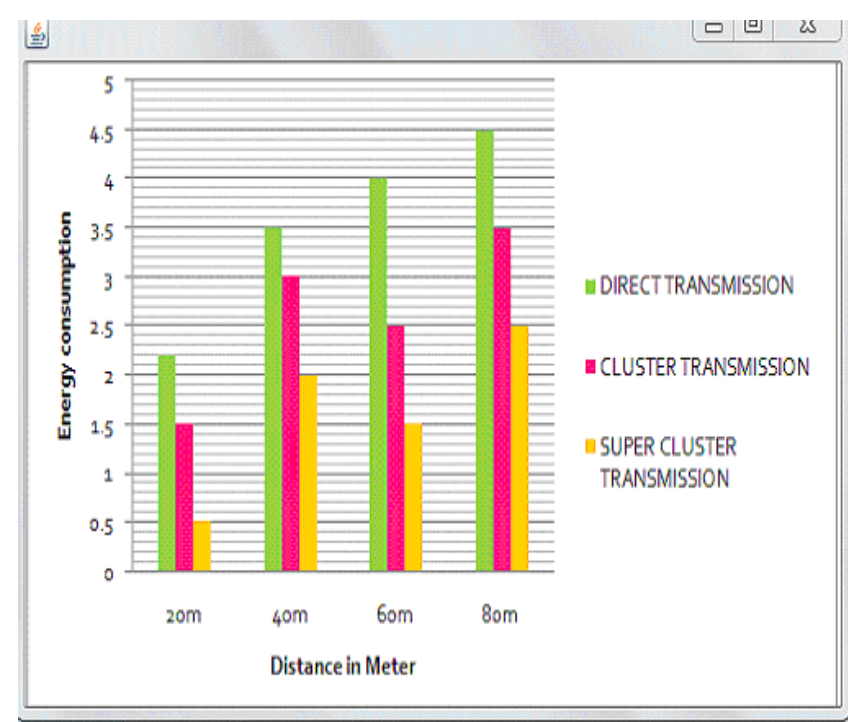

Figure 4. Energy consumption on varying distance.

In Figure 4 Energy consumption in Joule ( $n$ * 103 ) is taken as the $\mathrm{y}$ axis and three transmissions in $\mathrm{x}$ axis. Initially experimental area set up is 20X20 and energy consumption is measured. The area extends to $40 \mathrm{X} 40$, $60 \mathrm{X} 60,80 \mathrm{X} 80$ then energy consumption was measured. In all areas mentioned above, Super Cluster Formation consumes less energy than Direct Transmission and Cluster Formation as given in the graph.

\section{Conclusion}

The primary purpose of this paper is to prevent the Hotspot problem by using the Super Clustering mechanism. Clusters are formed by using the distance attribute. The proposed algorithm provides scalability and data Aggregation. Scalability is better when compared to the direct transmission method and Cluster Transmission method. Aggregation reduces the redundancy of data. The performance of the algorithm is evaluated through simulation. In the proposed method all nodes are taken into account by distance parameter. The future work is to change the cluster formation parameters so that cluster efficiency may be improved. Microsoft Word document.

\section{References}

1. Abdulla AEAA, Nishiyama H, Kato N. Extending the lifetime of wireless sensor networks: a hybrid routing algorithm. Comput Comm. 2012; 35:1056-63.
2. Yi S, Heo J, Cho Y, Hong J. Peach: Power-efficient and adaptive clustering hierarchy protocol for wireless sensor networks. Comput Comm. 2007; 30:2842-52.

3. Akkaya K, Younis M. A survey on routing protocols for wireless sensor networks. Ad Hoc Networks. 2005; 3(3):325-49.

4. Younis O, Fahmy S. Heed: a hybrid, energy-efficient, distributed clustering approach for ad hoc sensor networks. IEEE Transactions on Mobile Computing. 2004; 3(4):366-79.

5. Akyildiz, Su W, Sankarasubramaniam Y, Cayirci E. A survey on sensor networks. IEEE Communications Magazine. 2002; 40(8):102-14.

6. Toh C-K. Maximum battery life routing to support ubiquitous mobilecomputing in wireless ad hoc networks. IEEE Communications Magazine. 2001; 39(6):138-47.

7. Rodoplu V, Meng T. Minimum energy mobile wireless networks. IEEE J Sel Area Comm. 1999;17(8):1333-44.

8. Singh $S$, Woo M, Ragahavendra CS. Power-aware routing in mobile ad hoc networks. Proceedings of the 4th Annual ACM International Conference on Mobile Computing and Network, MobileCom ; 1998. New York, USA. p. 181-90.

9. Intanagonwiwat C, Govindan R, Estrin D. Directed diffusion: a scalable and robust communication paradigm for sensor networks. Proceedings of Sixth Annual ACM/ IEEE International Conference on Mobile Computing and Networking (MOBICOM); 2000 Aug. p. 56-67.

10. Heinzelman WB. Application-specific protocol architectures for wireless networks [PhD thesis] Massachusetts Institute of Technology. 2000 Jun.

11. Manjeshwar A and Agarwal DP. TEEN: a routing protocol for enhanced efficiency in wireless sensor networks. Proceedings of 1st International Workshop on Parallel and Distributed Computing Issues in Wireless Networks and Mobile Computing; 2001.

12. Haas ZJ, Halpern JY; Li L. Gossip-Based Ad Hoc Routing. Proceedings of the 19th Conference of the IEEE Communications Society (INFOCOM); 2002 Jun 23-27; New York, USA. p. 1707-16.

13. Kulik J, Heinzelman WR, Balakrishnan H. Negotiation based protocols for disseminating information in wireless sensor networks. Wirel Netw. 2002; 8:169-85.

14. Heinzelman W, Chandrakasan A, Balakrishnan H. An application-specific protocol architecture for wireless micro sensor networks. IEEE Transcation on Wireless Communication. 2002.

15. Lindsey S, Raghavendra CS. PEGASIS: Power Efficient Gathering in Sensor Information Systems. IEEE Aerospace Conference; 2002 Mar. p. 1-6.

16. Intanagonwiwat C, Govindan R, Estrin D, Heidemann J. Directed diffusion for wireless sensor networking.IEEE/ ACM Transactions on Networking. 2003; 2-16. 
17. Hassanein $\mathrm{H}$, Luo J. Reliable energy aware routing in wireless sensor networks dependability and security in sensor networks and systems. Second IEEE Workshop on Dependability and Security in Sensor Networks and Systems (DSSNS); 2006

18. Taheri H, Neamatollahi P, Younis OM, Naghibzadeh S, Yaghmaee $\mathrm{MH}$. An energy-aware distributed clustering protocol in wireless sensor networks using fuzzy logic. Ad Hoc Networks. 2012 Sept; 10(7):1469-81.

19. Shi L, Zhang B, Mouftah HT, Ma J. DDRP: An efficient datadriven routing protocol for wireless sensor networks with mobile sinks. Int J Comm Syst. 2013; 26(10):1341-55.
20. Biradar RV, Patil VC, Mudholkar RR, Sawant SR. Classification and comparison of routing protocols in wireless sensor networks. Ubiquitous Computing and Communication Journal. 2009; 4:704-11.

21. Lin $\mathrm{K}$ et al. EBMA: Energy Balancing cluster on based on the cellular topology. Future Generat Comput Syst. 2012; 28:446-56.

22. Ehsan A, Alireza P, Jalil PM. Fear: a fuzzy-based energyaware routing protocol for wireless sensor networks. Networking and Internet Architecture. 2011. 